\title{
AMENDMENTS
}

\section{Author Correction: Loss of ELF5-FBXW7 stabilizes IFNGR1 to promote the growth and metastasis of triple-negative breast cancer through interferon- $\boldsymbol{\gamma}$ signalling}

Snahlata Singh, Sushil Kumar, Ratnesh Kumar Srivastava, Ajeya Nandi, Gatha Thacker, Hemma Murali, Sabrina Kim, Mary Baldeon, John Tobias, Mario Andres Blanco, Rizwan Saffie (D), M. Raza Zaidi, Satrajit Sinha (D), Luca Busino (D), Serge Y. Fuchs (iD and Rumela Chakrabarti (D)

\section{Correction to: Nature Cell Biology https://doi.org/10.1038/s41556-020-0495-y, published online 13 April 2020}

In the version of this Article originally published, there were several errors or additional details needed in the text and figures. Fig. 1i,j have been replaced. The legend has been updated as well to “i,j, Haematoxylin and eosin stains of lung sections (i) and quantification of these results $(\mathbf{j})$ showed an increase in the incidence of lung metastatic nodules in lungs harvested from C3-T $\mathrm{T}^{+}$Elf5 $5^{+/-}$mice $(n=8$ for C3-T $\mathrm{T}^{+}$Elf5 $5^{++} ; n=8$ for $\mathrm{C} 3-\mathrm{T}^{+}$Elf5 ${ }^{+/-}$). Statistical significance was determined by Mann Whitney $U$-test." For Fig. 5c,d GFP labelled EpRas control and EpRas Elf5-OE cells were generated to track metastasis in whole lung images as small metastatic foci may be missed during sectioning. The authors repeated the experiment with these cells and increased the number of injected cells from 50,000 cells/ MFP to 500,000 cells/MFP to see metastasis. This change was also reflected in the legend for Fig. $5 \mathrm{c}$, d as "c, GFP images showing more micro metastatic foci in lungs of mice injected with control (CTRL) cells than in lungs of mice injected with Elf5-OE EpRas cells. Cells were labelled with GFP (lentiviral vector pLEX-hFL2iG was used) using standard method. d, Quantification of lung metastasis from c ( $n=4$ mice/group). For c,d, 500,000 cells/MFP were injected into the mice. White arrows show $\mathrm{GFP}^{+}$micro metastatic foci in whole-mount lung image." The scale bars in $5 \mathrm{c}$ also represent $500 \mu \mathrm{m}$ instead of $100 \mu \mathrm{m}$. Images in Figs. $6 \mathrm{f}, 7 \mathrm{~g}$ and quantification in Fig $7 \mathrm{c}$ have also been replaced. The scale bar in $7 \mathrm{~g}$ is also $70 \mu \mathrm{m}$ instead of $100 \mu \mathrm{m}$. Extended Data Figs. 1 and 8 have also been replaced, and their legends have been updated accordingly.

Additionally, several sentences were added to the Methods. In the subsection 'animal studies', part of the second paragraph has been edited to read "For the orthotopic tumour models, 70,000 EpRas cells per MFP of IFN- $\gamma$ - and control-treated cells were injected into the MFP of 5-week-old BALB/c mice for primary tumour analysis. For lung metastasis studies in Extended Data Fig. 8g, 250,000 GFP labelled cells were injected into MFP (using lentivirus method). After sacrificing mice, lungs were washed in PBS and then imaged under Leica dissection microscope using GFP filter. In the experiments involving tumour generation from Elf5 re-expressing EpRas cells, 50,000 cells per MFP were injected into 5-week-old BALB/c mice. For lung metastasis studies in Fig. 5c,d, 500,000 GFP labelled tumour cells of indicated group were injected into MFP (using lentivirus method). After sacrificing mice, lungs were washed in PBS and then imaged under Leica dissection microscope M80 using GFP filter." Finally, the following sentence was added to the acknowledgements: "We thank the Penn Vet Comparative Pathology Core for assistance with embedding, sectioning, consultation and slide evaluation. The Penn Vet Comparative Pathology Core is supported by the Abramson Cancer Center Support Grant (P30 CA016520). We thank A.J.P. Klein-Szanto (Fox Chase Cancer Center) for helpful discussions and consultation on primary and metastatic tumour samples."

Published online: 4 August 2021

https://doi.org/10.1038/s41556-021-00733-7

(C) The Author(s), under exclusive licence to Springer Nature Limited 2021

\section{Publisher Correction: p27 controls Ragulator and mTOR activity in amino acid-deprived cells to regulate the autophagy-lysosomal pathway and coordinate cell cycle and cell growth}

\author{
Ada Nowosad (D), Pauline Jeannot, Caroline Callot, Justine Creff, Renaud Thierry Perchey, Carine Joffre (D), \\ Patrice Codogno D, Stephane Manenti and Arnaud Besson (D)
}

Correction to: Nature Cell Biology https://doi.org/10.1038/s41556-020-0554-4, published online 17 August 2020

In the version of this Article originally published, there was an error in Fig. 5c. The fourth symbol from the right in the HA-L1 row should be a plus instead of a minus (row should read ' -++-+-++ '). The error has been corrected.

Published online: 27 July 2021

https://doi.org/10.1038/s41556-021-00741-7

(C) The Author(s), under exclusive licence to Springer Nature Limited 2021 